BMJ Open Sport \& Exercise Medicine

\title{
Feasibility of a postacute structured aerobic exercise intervention following sport concussion in symptomatic adolescents: a randomised controlled study
}

\author{
Rachel Micay, Doug Richards, Michael G Hutchison
}

\begin{abstract}
To cite: Micay R, Richards D, Hutchison MG. Feasibility of a postacute structured aerobic exercise intervention following sport concussion in symptomatic adolescents: a randomised controlled study. BMJ Open Sport \& Exercise Medicine 2018;4:e000404. doi:10.1136/ bmjsem-2018-000404
\end{abstract}

Accepted 13 June 2018

\section{ABSTRACT}

Objective The utility of structured exercise for rehabilitation purposes early in the postacute phase (ie, beyond the initial 24-48 hours of advised rest) following sport-related concussion (SRC) remains largely unexplored. This study examined the feasibility of implementing a standardised aerobic exercise (AE) intervention in the postacute stage of SRC recovery in a sample of adolescent students with SRC compared with usual care.

Methods Symptomatic adolescents with SRC were randomised to one of two groups: Aerobic Exercise $(n=8)$ or Usual Care $(n=7)$. The AE intervention, beginning on day 6 postinjury, comprised eight sessions with progressive increases in intensity and duration on a cycle ergometer. Usual care consisted of rest followed by physician-advised progressions in activity levels in an unsupervised setting. All participants were evaluated by physician at weeks 1, 2, 3 and 4 postconcussion. Outcome measures included: (1) Intervention feasibility: symptom status pre-post exercise sessions and completion of intervention and (2) Clinical recovery: symptom status at weeks 1, 2, 3 and 4 postinjury and medical clearance date.

Results All participants completed the exercise sessions as part of the $A E$ intervention and symptom exacerbation was not associated with any exercise session. The AE group experienced greater symptom resolution compared with the Usual Care Group across the recovery timeline. Conclusion A structured AE protocol appears to be safe and feasible to administer in the postacute stage of SRC recovery in adolescents and should be explored as part of a full Phase III Clinical Trial.

\section{(C) Author(s) (or their} employer(s)) 2018. Re-use permitted under CC BY-NC. No commercial re-use. See rights and permissions. Published by BMJ.

Faculty of Kinesiology and Physical Education, University of Toronto, Toronto, Ontario, Canada

\section{Correspondence to} Rachel Micay; rachel.micay@ mail.utoronto.ca

\section{INTRODUCTION}

Historically, guidelines for the clinical management of sport-related concussion (SRC) have advised physical and cognitive rest until the achievement of asymptomatic status. ${ }^{12}$ While this postinjury rest period was previously assumed to be beneficial, it lacks the support of empirical evidence, and there is no consensus as to appropriate nature or duration. Conversely, the administration of low-to-moderate aerobic

\section{What are the new findings?}

- Postacute structured aerobic exercise (AE), beginning on day 6 postinjury, appears to be both safe and feasible to administer to symptomatic adolescent patients with sport-related concussion.

- The eight-session structured AE intervention implemented in this study was not associated with any exacerbation of symptoms and/or prolonged time to medical clearance compared with 'usual care'

- Structured AE appears to be associated with faster resolution of symptoms severity compared with 'usual care'.

How might it impact on clinical practice in the near future?

- Findings support the emerging paradigm shift in concussion management towards active rehabilitation rather than rest.

- Clinicians now have empirical evidence to support the safe prescription of light-to-moderate aerobic exercise as an intervention in the early stages of recovery following sport-related concussion (SRC).

- Results support the increasing obsolescence of rest in the clinical management of SRC.

exercise (AE), a well-established mechanism of neuronal regeneration following a traumatic event, ${ }^{34}$ has demonstrated efficacy in expediting recovery time and improving functional outcomes following various injuries to the central nervous system including stroke, ${ }^{56}$ whiplash $^{7-9}$ and low back pain. ${ }^{10-12}$

A growing body of evidence highlights the benefits of structured AE in facilitating recovery across the traumatic brain injury (TBI) spectrum. For example, low-to-moderate $\mathrm{AE}$, administered as early as 2 days postinjury in rodents, has been associated with significant downregulation of apoptotic factors in the neurons of the tissue surrounding 
the site of the injury ${ }^{13}$ and significant increases to cell proliferation and neurogenesis. ${ }^{14}$ In humans, structured $\mathrm{AE}$ has demonstrated efficacy in facilitating the recovery of individuals with persistent postconcussion symptoms. For example, Leddy and colleagues (2010) demonstrated that the implementation of a controlled, graded $\mathrm{AE}$ programme involving treadmill exercise (5-6 days per week at $80 \%$ of the individual's predetermined symptom exacerbation threshold) led to a significantly more rapid recovery and significantly fewer postconcussion symptoms in a cohort of patients with persistent symptoms compared with rest. ${ }^{15}$

Despite these promising advancements in support of active rehabilitation, no study to date has explored the efficacy of a structured AE intervention in humans within the acute stages of the injury. The Concussion in Sport Group's most recently published guidelines acknowledge that rest beyond the initial 24-48 hours of SRC is not beneficial to the recovery process. ${ }^{16}$ As such, the ideal nature and duration of postconcussion activity must now be defined. The purpose of this study was to determine the feasibility of implementing a standardised $\mathrm{AE}$ intervention in the postacute stage of SRC recovery in adolescent athletes. Specifically, we evaluated participant safety and logistics, and estimated the treatment effect of the standardised $\mathrm{AE}$ intervention on facilitating clinical recovery following SRC compared with usual care.

\section{METHODS}

\section{Participant recruitment and randomisation}

Eligible participants included adolescent athletes (aged 14-18 years) with a physician-diagnosed SRC who were symptomatic at day 5 postinjury, which was operationally defined as a symptom severity score of greater than five on the Post-Concussion Symptom Scale (PCSS) of the SCAT3. Symptomatic participants were then randomised into either the Usual Care Group (UCG) or the Exercise Group (EXG) using a computer-based randomisation algorithm. Informed consent was obtained for all participants, in addition to parental consent for participants under the age of 16. Ethical approval for this study was granted by the University of Toronto Health Sciences Research Ethics Board and the trial was registered on a public database (clinicaltrials.gov, protocol reference \#33459).

UCG participants were directed through the existing six-stage progression of activity by their attending sport-medicine physician, consistent with the Berlin 2016 guidelines $^{16}$ and were assessed at weeks 1, 2, 3 and 4 postinjury on measures of clinical recovery, including return-to-play (RTP) status and PCSS symptom severity. RTP status was determined by a sports medicine physician who was blinded to the participant's group placement. EXG participants were directed through a standardised $\mathrm{AE}$ intervention beginning on day 6 postinjury while concurrently being directed through the six-stage activity progression by their attending physician. These participants were assessed using the same measures and at the same time intervals as the UCG participants.

\section{Standardised AE intervention}

TheAE intervention consisted of eightsessions proceeding in a stepwise fashion with respect to duration and intensity using the Velotron Racermate Pro stationary cycle ergometer (RacerMate, Washington, USA), which was digitally connected to a heart rate monitor (Polar Electro Oy, Kemple, Findland) to ensure that participants maintained an appropriate exercise intensity corresponding with their age and stage in the recovery timeline. The first session was $10 \mathrm{~min}$ in duration and exercise intensity was set to correspond with $50 \%$ of the participant's age-predicted maximal heart rate (HR). This intensity was maintained at the next session, however, the session was increased to $20 \mathrm{~min}$ in duration. All subsequent sessions were $20 \mathrm{~min}$ in duration, and exercise intensity increased by $5 \%$ of the individual's age-predicted maximal HR per session until $70 \%$ was reached, which was maintained for the final three sessions. All sessions began with a 5 min warmup period and concluded with a 5 min cool-down period on the cycle ergometer. Exercise occurred on two consecutive days, followed by 1 day of rest, for a total of 11 days.

PCSS scores were collected before and after each exercise session and symptoms were monitored throughout exercise using a prepared script. Symptom exacerbation was defined as an increase in the PCSS symptom severity score by 3 or more, based on the operational definition of exercise-induced symptom exacerbation previously provided by Willer and Leddy. ${ }^{17}$ If symptoms worsened at any point during exercise, the session was terminated and the same duration and intensity of exercise would be attempted at the subsequent session. If a participant's symptoms became exacerbated again at the subsequent session, their participation in the intervention portion of the study was terminated. Once participants in the EXG achieved asymptomatic status, they continued to progress through the protocol until completing all eight sessions or until they received full medical clearance to RTP.

\section{Outcome measures}

The AE intervention was determined to be feasible if: (1) symptom did not become exacerbated during or immediately after exercise compared with pre-exercise levels and (2) EXG participants were able to complete the entire AE intervention. The efficacy of the intervention was evaluated by symptom status and time to medical clearance (in days) compared with usual care. Time to medical clearance (in days) was determined by reviewing each participant's electronic medical record for their RTP status, while resolution in symptom severity across the recovery timeline was determined based on self-reported symptom severity scores on the PCSS, collected on a weekly basis for a full month postinjury. 


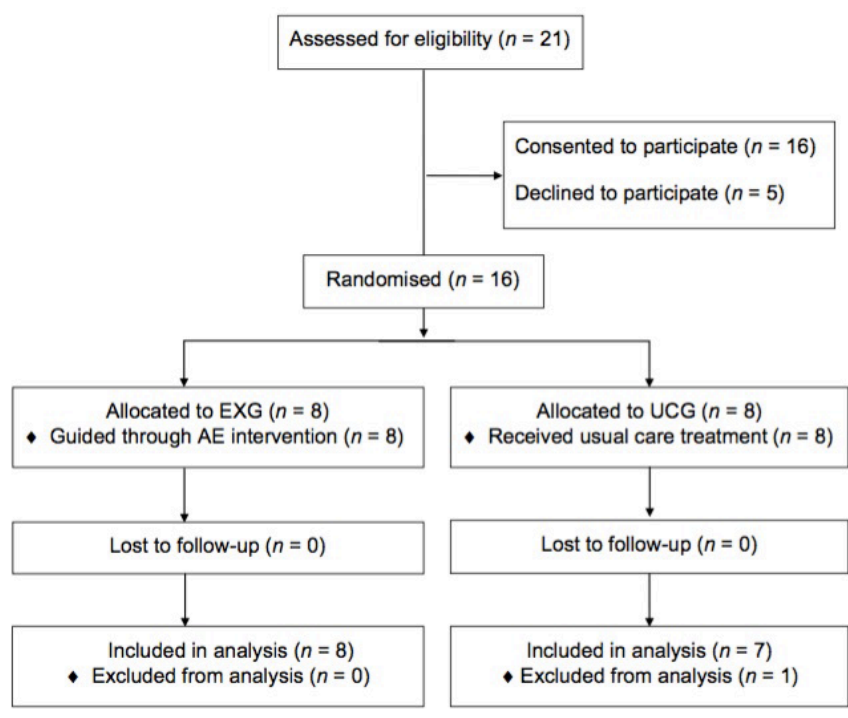

Figure 1 Participant recruitment and randomisation. EXG, Exercise Group; UCG, Usual Care Group.

\section{Statistical analysis}

A series of independent t-tests were used to evaluate between-group differences in demographic and injury characteristics, specifically age, height, mass, prior concussions, pre-existing medical conditions, preinjury activity levels and initial symptom burden. To investigate the feasibility of the $\mathrm{AE}$ intervention within the EXG participants, a series of paired-sample t-tests were completed to determine differences in PCSS symptom severity scores pre-exercise and postexercise at each session. We also calculated the percentage of individuals lost to follow-up. To examine the efficacy of the AE intervention, a multiple linear regression analysis was used to examine between-group differences in overall time to medical clearance (days), while adjusting for acute symptom severity. Paired t-tests were also completed for both groups to determine within-group differences in the resolution of symptom severity across the recovery timeline.

\section{RESULTS}

\section{Participant recruitment}

Recruitment of participants occurred between January and June, 2017 at the University of Toronto's David L. Macintosh Sport Medicine Clinic. Figure 1 illustrates the recruitment and flow of participants through the trial. A total of 21 patients meeting the eligibility criteria consented to be contacted by a member of the research team to learn more about the study. Of these 21 patients, $\mathrm{n}=16(76 \%)$ of them went on to participate in the study. Using a predetermined randomisation schedule generated on an electronic randomisation platform, eight participants each were allocated into the EXG and UCG. None of the participants in either group were lost to attrition, although one UCG participant was removed from
Table 1 Participant characteristics (mean and SD) stratified by group randomisation

\begin{tabular}{llll}
\hline & $\begin{array}{l}\text { Exercise } \\
\text { Group (n=8) }\end{array}$ & $\begin{array}{l}\text { Usual Care } \\
\text { Group (n=7) }\end{array}$ & P values \\
\hline Age (years) & $15.8 \pm 1.2$ & $15.6 \pm 1.0$ & 0.76 \\
\hline Height (cm) & $179.7 \pm 8.3$ & $176.7 \pm 3.6$ & 0.40 \\
Mass (kg) & $74.9 \pm 12.8$ & $82.0 \pm 11.0$ & 0.27 \\
$\begin{array}{l}\text { Number of previous } \\
\text { concussions (median } \\
\text { and range) }\end{array}$ & $0(0-2)$ & $0(0-2)$ & 0.58 \\
$\begin{array}{l}\text { Number of pre-existing } \\
\text { medical conditions } \\
\text { (median and range) }\end{array}$ & $1(0-2)$ & $1(0-2)$ & 0.18 \\
$\begin{array}{l}\text { Weekly leisure activity } \\
\text { score }\end{array}$ & $79.9 \pm 7.4$ & $78.4 \pm 27.2$ & 0.90 \\
$\begin{array}{l}\text { Acute PCSS symptom } \\
\text { severity score }\end{array}$ & $32.0 \pm 13.0$ & $24.4 \pm 18.7$ & 0.37 \\
\hline
\end{tabular}

PCSS, Post-Concussion Symptom Scale.

the dataset as this individual was the sole female participant of the study. As such, the study sample comprised 15 male participants with SRC $(n=15)$, all of whom were symptomatic at day 5 postinjury (PCSS symptom severity score of $>5)$.

\section{Participant demographics}

Independent t-tests revealed no significant differences between groups with respect to age, height, mass, concussion history, the presence of pre-existing medical conditions (including anxiety, depression, migraines and/or attention deficit hyperactivity disorder), preinjury exercise levels (based on responses to the Godin Leisure-Time Exercise Questionnaire) and the number/severity of symptoms on the PCSS at day 5 postinjury. A detailed description of these demographic variables as well the results from a series of independent t-tests between groups can be found in table 1 .

\section{Safety of the AE intervention}

The structured AE intervention was not associated with symptom exacerbation at any point during or immediately after any of the exercise sessions. Figure 2 illustrates that average postsession PCSS symptom severity scores were generally lower than average presession symptom severity scores, although results from a series of paired t-test found that this difference was only statistically significant at Session $1(t(7)=3.2, \mathrm{p}=0.02)$.

\section{Aerobic exercise intervention efficacy}

\section{Time to medical clearance}

Independent t-tests did not identify a significant betweengroup difference in mean time to medical clearance, which was $36.1 \pm 18.5$ days for the EXG and 29.6 \pm 15.8 days for the UCG $(t(13)=0.03 ; \mathrm{p}=0.87)$. For exploratory purposes, multiple linear regression analysis was used to examine between-group differences in time to medical clearance while controlling for acute symptom severity 


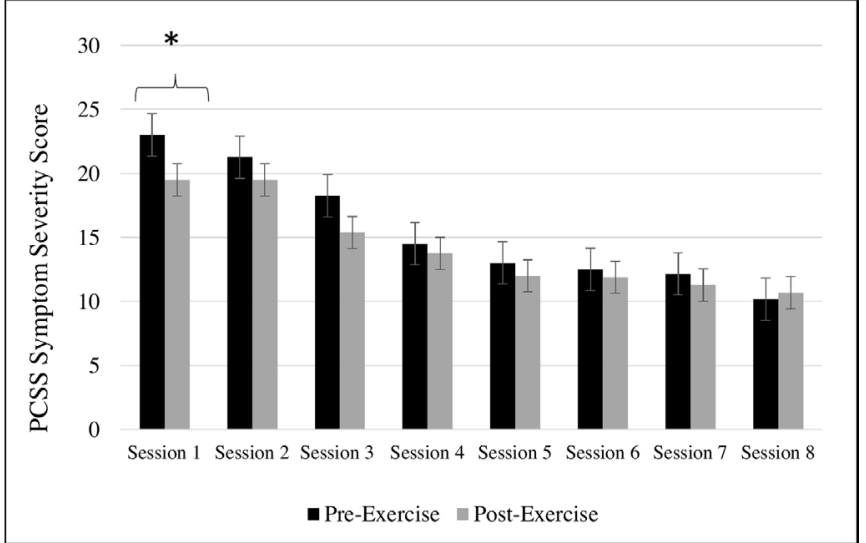

Figure 2 Symptom severity scores for the EXG preexercise and post-exercise sessions (*indicates statistical significance, $p<0.05)$. AE, aerobic exercise; PCSS, PostConcussion Symptom Scale.

(ie, symptom severity at day 5 postinjury). The model overall was not statistically significant $(F(2,14)=3.2$, $\left.\mathrm{p}=0.08), R^{2}=0.35\right)$ and the between-group difference was also not found to be significant $(\mathrm{p}=0.82)$. However, there was a significant association identified between time to medical clearance and acute symptom severity (ie, PCSS symptom severity at day 5 postinjury), which yielded a beta coefficient score of $0.57(\mathrm{p}=0.04)$.

\section{Symptom resolution across the recovery timeline}

The resolution in mean PCSS symptom severity scores for both groups across the first month of recovery is illustrated in figure 3. On visual inspection, it appears that the resolution in symptom severity was noticeably more pronounced in the EXG compared with the UCG, particularly between Weeks 1 and $3(15.8 \pm 1.9$ vs $6.7 \pm 4.1$ points, respectively) and Weeks 1 and $4(18.8+4.9$ points

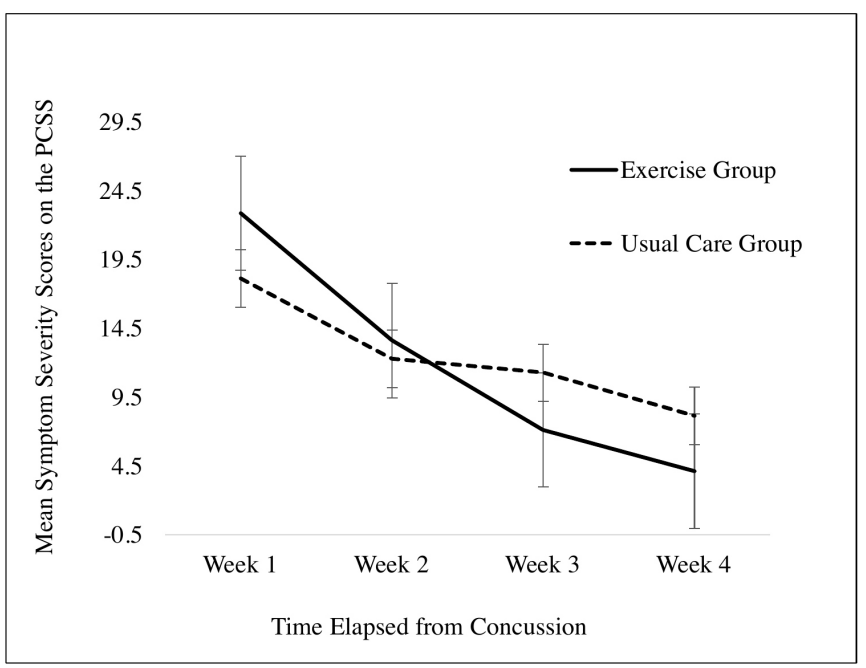

Figure 3 Mean symptom severity scores reported on the PCSS component of the SCAT3 across the recovery timeline (error bars: \pm 1 SE). PCSS, Post-Concussion Symptom Scale; $\mathrm{SRC}$, sport-related concussion. vs $10.0 \pm 6.1$ points, respectively) postinjury. Results from a series of paired t-tests demonstrate that the EXG experienced significant within-group differences in symptom resolution between Weeks 1 and $2(\mathrm{t}(7)=3.4, \mathrm{p}=0.01)$, Weeks 2 and $3(\mathrm{t}(7)=2.6, \mathrm{p}=0.04)$, Weeks 1 and $3(\mathrm{t}(7)=6.4$, $\mathrm{p}<0.01)$ and Weeks 1 and $4(\mathrm{t}(7)=7.8, \mathrm{p}<0.01)$ postconcussion. Within the UCG group, significant differences in resolution in symptom severity were observed between Weeks 1 and $4(\mathrm{t}(6)=2.5, \mathrm{p}<0.05)$ and Weeks 3 and 4 $(\mathrm{t}(6)=3.0, \mathrm{p}=0.02)$ postconcussion.

\section{DISCUSSION}

This is the first study to report the feasibility and preliminary efficacy of a structured $\mathrm{AE}$ intervention in adolescents in the postacute days following SRC. Preliminary findings from the study are promising as we have provided evidence that structured $\mathrm{AE}$ initiated within the first week of concussion among symptomatic patients is feasible and safe to administer, given that symptom status did not worsen during, of after, each exercise session and all participants were able to complete the intervention protocol over the 11 days. Furthermore, the administration of a $\mathrm{AE}$ intervention appears to be associated with a reduction in symptom severity scores across the first 4 weeks post injury.

\section{Symptom resolution}

In the current study, we found that the EXG participants reported a significant reduction in symptom severity scores compared with the usual care participants. Specifically, EXG participants experienced significant decrease in symptom severity between Weeks 1 and 3 postinjury, while their UCG counterparts did not achieve significant resolution until Week 4 postinjury. While our limited sample size lacked the statistical power to detect between-group differences in symptom scores at specific times across SRC recovery, exploratory analyses with an effect size of 0.80 indicate a sample size of 30 participants (15 in each group) is required to identify between-group differences in symptom status across the 4 weeks postinjury.

Our results from the present study align with previous research examining the effect of rest on symptom resolution in adolescents following SRC. A randomised control trial by Thomas and colleagues ${ }^{18}$ found that $50 \%$ of adolescent patients with mTBI undergoing 5 days of strict rest following concussion took approximately 3 days longer to achieve symptom resolution compared with patients who underwent 24-48 hours of rest followed by a gradual return to activities. Moreover, the strict rest patients also reported a significantly higher overall symptom severity scores over the 10-day follow-up period compared with those who underwent the active rehabilitation protocol. ${ }^{18}$ Collectively, these findings provide further evidence to a growing body of literature suggesting that active rehabilitation may be associated with more desirable outcomes compared with rest. ${ }^{181920}$ 


\section{Time to medical clearance}

Although we did not identify earlier time to medical clearance for the EXG compared with UCG, a significant correlation was identified between acute symptom severity (ie, PCSS symptom severity score at day 5 postinjury) and overall time to medical clearance across both groups. Specifically, individuals reporting a higher initial symptom severity score took significantly longer to obtain medical clearance to return to their preinjury participation levels. Thus, it appears acute symptom burden may be a meaningful predictor of time to medical clearance, regardless of group placement. This finding is supported by a recent systematic review by Iverson and colleagues, ${ }^{21}$ which reported that acute and subacute symptoms is the most consistent predictor of prolonged recovery from concussion across the literature. $^{21}$ These findings highlight the need for future research studies to control for initial symptom burden as part of the group randomisation process.

\section{Future directions}

The primary purpose of this pilot study was to determine the practical and clinical feasibility of implementing this type of protocol, thereby providing evidence to warrant a larger scale clinical trial. Future studies should aim to recruit a statistically powered sample size. Based on exploratory sample size calculation analyses, this would be achieved with a sample size of $n=30$. Our study sample was limited to male participants; future studies should also consider a sample size large enough to explore potential differences between males and females giving the growing body of literature identifying differential responses postconcussion. $^{22-24}$ Furthermore, while the clinical decision to medically clear a patient is well established (ie, the achievement of asymptomatic status following a graded exertional protocol), previous research has demonstrated a great degree of variability in individual physician practices with respect to assessment, management and clearance following concussion. ${ }^{25}$ Also, there are some practical issues that should be acknowledged with using medical clearance as an outcome measure, such as physician availability and scheduling, patient availability,and urgency. Future studies may consider limiting the number of physicians to ensure consistency in the clinical management of participants or using an outcome measure other than time to medical clearance as a marker of clinical recovery following concussion, such as time to return to light AE (stage 2 of the existing RTP guidelines). ${ }^{16}$ It may also be beneficial for future studies to assess the inter-rater reliability of physicians with respect to their return-to-school or RTP decisions. Additionally, given the objective of assessing the efficacy of an $\mathrm{AE}$ intervention, it is recommended that future studies implement activity logs and/or wearable activity trackers to monitor compliance among participants and control for any unsupervised physical activity performed outside of the AE intervention setting. Lastly, future studies should consider acquiring additional symptom status evaluations given the timeline of the neurometabolic cascade of $\mathrm{mTBI},{ }^{26}$ which is correlated with the presentation of subjective symptoms following SRC. ${ }^{27}$ This would allow for a more precise understanding of how symptoms resolve in the immediate stages following injury and how this is affected by the administration of $\mathrm{AE}$ within the adolescent population.

\section{CONCLUSION}

This study is the first randomised control trial to demonstrate that a standardised, graded AE intervention in the low-to-moderate intensity range is safe and feasible to administer in adolescents within the postacute stage of SRC recovery. Given the novelty of this protocol and the conservative approach taken in its design, future studies building on it should explore a wider range of exercise intensities and modalities in order to determine the optimal parameters for facilitating recovery. Future studies should also attempt to initiate exercise at an earlier stage in the recovery timeline, particularly in light of the most recently published guidelines from the Concussion in Sport Group, which acknowledge the lack of evidence to suggest that rest beyond the initial 24-48 hours of injury is of any benefit to the recovery process. $^{16}$

Acknowledgements The authors would like to acknowledge the patients, family, staff and physicians of the David L. MacIntosh Sport Medicine Clinic at the University of Toronto for their participation and continuous support of this study, without which this work would not be possible. We would also like to thank Rob Rupf and Ming Chang Tsai for their technical expertise in using the Velotron Racermate stationary cycle ergometer and corresponding software.

Contributors RM recruited participants, implemented and monitored data collection, administered the exercise intervention, processed the data, executed the statistical analyses and drafted and revised the paper. MGH contributed to the design of the intervention and data collection protocol, supervised participant recruitment and data collection, assisted in data analysis, revised the paper and facilitated the funding of this study. DR generated the theoretical basis for this study, contributed to planning and coordinating its implementation and supervised all aspects of clinical operations.

Funding This study was funded by the Steve Moore Foundation (Toronto, 0N, Canada), grant number 501044.

Competing interests None declared.

Patient consent Obtained.

Ethics approval University of Toronto Research Ethics Board.

Provenance and peer review Not commissioned; internally peer reviewed.

Open access This is an Open access article distributed in accordance with the Creative Commons Attribution Non Commercial (CC BY-NC 4.0) license, which permits others to distribute, remix, adapt, build upon this work non-commercially, and license their derivative works on different terms, provided the original work is properly cited, appropriate credit is given, any changes made indicated, and the use is non-commercial. See: http://creativecommons.org/licenses/by-nc/4.0/.

\section{REFERENCES}

1. McCrory P, Meeuwisse W, Johnston K. Consensus statement on concussion in sport: the 3rd International conference on concussion in sport held in Zurich, November 2008. Br J Sports Med 2009;43(Suppl 1):i76-i84.

2. McCrory P, Meeuwisse WH, Aubry M, et al. Consensus statement on concussion in sport: the 4th International conference on concussion in sport held in Zurich, November 2012. Br J Sports Med 2013;47:250-8. 
3. Neeper SA, Gómez-Pinilla F, Choi J, et al. Physical activity increases mRNA for brain-derived neurotrophic factor and nerve growth factor in rat brain. Brain Res 1996;726:49-56.

4. Cotman CW, Berchtold NC. Exercise: a behavioral intervention to enhance brain health and plasticity. Trends Neurosci 2002;25:295-301.

5. Albert SJ, Kesselring J, stroke Nof. Neurorehabilitation of stroke. $J$ Neurol 2012;259:817-32.

6. Cumming TB, Collier J, Thrift AG, et al. The effect of very early mobilisation after stroke on psychological well-being. $J$ Rehabil Med 2008:40:609-14.

7. Teasell RW, McClure JA, Walton D, et al. A research synthesis of therapeutic interventions for whiplash-associated disorder (WAD): part 4 - noninvasive interventions for chronic WAD. Pain Res Manag 2010;15:313-22.

8. Schnabel M, Ferrari R, Vassiliou T, et al. Randomised, controlled outcome study of active mobilisation compared with collar therapy for whiplash injury. Emerg Med J 2004;21:306-10.

9. Söderlund A, Olerud C, Lindberg P. Acute whiplash-associated disorders (WAD): the effects of early mobilization and prognostic factors in long-term symptomatology. Clin Rehabil 2000;14:457-67.

10. Dahm KT, Brurberg KG, Jamtvedt G. Advice to rest in bed versus advice to stay active for acute low-back pain and sciatica. Chichester, UK: John Wiley \& Sons, Ltd, 1996:1-3.

11. Hagen KB, Jamtvedt G, Hilde G, et al. The updated cochrane review of bed rest for low back pain and sciatica. Spine 2005;30:542-6.

12. Waddell G, Feder G, Lewis M. Systematic reviews of bed rest and advice to stay active for acute low back pain. $\mathrm{Br} J$ Gen Pract 1997:47:647-52.

13. Itoh $\mathrm{T}$, Imano $\mathrm{M}$, Nishida $\mathrm{S}$, et al. Exercise increases neural stem cell proliferation surrounding the area of damage following rat traumatic brain injury. J Neural Transm 2011;118:193-202.

14. Jacotte-Simancas A, Costa-Miserachs D, Coll-Andreu M, et al. Effects of voluntary physical exercise, citicoline, and combined treatment on object recognition memory, neurogenesis, and neuroprotection after traumatic brain injury in rats. $J$ Neurotrauma 2015;32:739-51.

15. Leddy JJ, Kozlowski K, Donnelly JP, et al. A preliminary study of subsymptom threshold exercise training for refractory postconcussion syndrome. Clin J Sport Med 2010;20:21-7.
16. McCrory P, Meeuwisse W, Dvořák J. Consensus statement on concussion in sport-the 5th international conference on concussion in sport held in Berlin, October 2016. 2017;51:838-47.

17. Leddy JJ, Willer B. Use of graded exercise testing in concussion and return-to-activity management. Curr Sports Med Rep 2013;12:370-6.

18. Thomas DG, Apps JN, Hoffmann RG, et al. Benefits of strict rest after acute concussion: a randomized controlled trial. Pediatrics 2015;135:213-23.

19. Leddy JJ, Cox JL, Baker JG, et al. Exercise treatment for postconcussion syndrome: a pilot study of changes in functional magnetic resonance imaging activation, physiology, and symptoms. $J$ Head Trauma Rehabil 2013;28:241-9.

20. Gagnon I, Galli C, Friedman D, et al. Active rehabilitation for children who are slow to recover following sport-related concussion. Brain Inj 2009;23:956-64.

21. Iverson GL, Gardner AJ, Terry DP, et al. Predictors of clinical recovery from concussion: a systematic review. $\mathrm{Br} J$ Sports Med 2017;51:941-8.

22. Di Battista AP, Rhind SG, Baker AJ, et al. An investigation of neuroinjury biomarkers after sport-related concussion: from the subacute phase to clinical recovery. Brain Inj 2018;32:575-82.

23. Hutchison MG, Mainwaring L, Senthinathan A, et al. Psychological and physiological markers of stress in concussed athletes across recovery milestones. J Head Trauma Rehabil 2017;32:E38-48.

24. Ono KE, Burns TG, Bearden DJ, et al. Sex-based differences as a predictor of recovery trajectories in young athletes after a sportsrelated concussion. Am J Sports Med 2016;44:748-52.

25. Stern RA, Seichepine D, Tschoe $C$, et al. Concussion care practices and utilization of evidence-based guidelines in the evaluation and management of concussion: a survey of New England emergency departments. J Neurotrauma 2017;34:861-8.

26. Giza CC, Hovda DA. The new neurometabolic cascade of concussion. Neurosurgery 2014;75(Suppl 4):S24-33.

27. Henry LC, Tremblay S, Boulanger Y, et al. Neurometabolic changes in the acute phase after sports concussions correlate with symptom severity. J Neurotrauma 2010;27:65-76. 